\title{
Fuentes estadísticas para analizar el sector de la restauración en España*
}

\author{
Laura Cabiedes Miragaya
}

Universidad de Oviedo

lcabie@uniovi.es

\section{Lluís Miret-Pastor}

Universitat Politècnica de València

luimipas@esp.upv.es

Recepción: 27-07-2017

Aceptación: 08-04-2018

Publicación: 16-10-2018

\section{Resumen}

La restauración es una parte fundamental del turismo. Sin embargo, existen pocos trabajos cuantitativos ya que es un sector difícil de estudiar. El objetivo de este artículo es analizar las posibilidades de análisis del sector de la restauración en España con apoyo en las principales fuentes estadísticas. Con este fin, se describen y comparan las bases de datos disponibles para analizar el sector tanto desde la perspectiva de la demanda como de la oferta. El artículo concluye con algunas sugerencias a efectos de qué estadísticas conviene utilizar y qué medidas sería deseable que fuesen adoptadas para mejorar la información estadística.

Palabras clave: restauración; servicios de comidas y bebidas; Horeca; fuentes estadísticas; comer fuera del hogar

* Este artículo ha sido elaborado en el marco de dos proyectos de investigación financiados por el Plan Nacional de I+D+i del MEC, de cuyo equipo es miembro la primera de los autores. Se trata de los proyectos titulados «La alimentación fuera del hogar en Europa: un análisis comparado de los modelos alimentarios extradomésticos en España y Reino Unido» (dirigido por Cecilia Díaz Méndez, Universidad de Oviedo. Ref. CSO2012-31904) y «Alimentación y estructura social. Análisis de las desigualdades sociales alimentarias en España» (dirigido por Isabel García Espejo, Universidad de Oviedo. Ref: CSO2015-68434-R). 
Abstract. Statistical sources for the analysis of the food service sector in Spain

Food and beverage service activities are a key part of tourism. However, there are few quantitative works as it is a difficult sector to study. This paper aims to explore the possibilities of analysing the food service sector in Spain using the main statistical sources. For this purpose, the available databases have been described and compared. Databases with information on both the demand and the supply side have been the object of study. The article concludes with some suggestions regarding the prioritization of statistics in order to study the sector and which measures would be desirable to improve the available statistical information.

Keywords: food service; catering services; Horeca; statistical sources; eating out

\section{Sumario}

\section{Introducción}

2. Dificultades para definir y cuantificar el sector de la restauración en España

3. La dispersión de estadísticas y las principales bases de datos disponibles
4. Una aproximación al sector de la restauración a través de la estadística oficial

5. Conclusiones

Referencias bibliográficas

\section{Introducción}

El sector de la restauración desempeña un papel fundamental en la economía española en general y en el sector turístico en particular. En el año 2016 la producción del sector en su conjunto (restaurantes, cafeterías, bares y comedores colectivos) ascendió a casi 93.000 millones de euros, lo que representa alrededor del 5\% de la producción nacional total y más de 1,2 millones de personas empleadas (Figuerola, 2015; Figuerola et al., 2017). Además, dado que la producción correspondiente a servicios de alojamiento ascendió a casi 27.000 millones de euros en el mismo año, se puede afirmar que prácticamente el $78 \%$ del sector de la hostelería tiene como actividad principal la prestación de servicios de comidas y bebidas en los establecimientos mencionados. No obstante, hasta hace unos 30 años apenas se tenía conocimiento de su importancia en la economía de nuestro país. Los datos estadísticos se limitaban prácticamente a los publicados en los anuarios de la Dirección General de Turismo, donde, además, se excluían los entonces llamados café-bares y los escasos estudios específicos del sector se limitaban al número de establecimientos y su categoría, sin entrar en magnitudes de empleo o producción (Guerra, 2009).

En general, la información sobre los servicios en España ha estado en desventaja respecto a la disponible para otras actividades económicas, y ha presentado carencias y problemas estadísticos, como la escasez de medios para la obtención de datos oficiales sobre el sector o su propia naturaleza (el carácter intangible y multidimensional de numerosos servicios), aspectos que dificul- 
tan su definición y medición estadística, en particular la cuantificación de los atributos cualitativos (Cuadrado Roura, 2003). Sin embargo, las estadísticas oficiales relacionadas con el turismo comenzaron a experimentar un cambio notorio desde finales de los años 90, momento en el que, por ejemplo, el Instituto Nacional de Estadística (INE) empezó a realizar encuestas cada vez más frecuentes y completas, de modo que, partiendo de tan solo algunas referidas a aspectos económicos para hoteles y restauración con periodicidad cuatrienal o quinquenal, procederá a facilitar información anual para el conjunto de actividades relacionadas con el turismo (Castro, 2000). No obstante, en muchas ocasiones la información disponible no es útil para analizar específicamente el sector de la restauración en España, en gran medida porque el objetivo declarado de muchas de las bases de datos oficiales es facilitar información rigurosa sobre el sector turístico, y en este contexto o bien se ha dado clara prioridad a los servicios de alojamiento frente a los de restauración o bien estos resultan englobados con los primeros bajo el paraguas de hostelería, lo que dificulta un análisis diferenciado del sector.

El hecho de que el vacío estadístico señalado fuese dotando a la restauración de la imagen de hermana pobre de la hostelería y el turismo llegó a ser en parte compensado por el primer estudio económico sobre restaurantes realizado en España. Se trata de un informe llevado a cabo por M. Figuerola a finales de los años 80, editado por la FEHR (Federación Española de Hostelería), en colaboración con American Express. En él las cifras manejadas pusieron de manifiesto que, frente a la percepción generalizada, los restaurantes superaban en volumen de negocio y en nivel de empleo a las empresas hoteleras (García y Pin, 2010; Guerra, 2009), lo que situaba a la restauración como el subsector más importante del conjunto de la hostelería. De modo similar, la publicación en 1991 de una macroencuesta realizada por el INE sobre la estructura empresarial del sector de la restauración sorprendió en la medida en la que los café-bares, hasta entonces apenas estudiados, resultaron ser el grupo empresarial más relevante de la restauración en volumen de ventas y nivel de empleo (Guerra, 2009). A pesar de lo indicado previamente, no es fácil encontrar actualmente información estadística referida específicamente al sector de la restauración en España.

El objetivo de este artículo es explorar las posibilidades de análisis del sector de la restauración en España con apoyo en las principales bases de datos disponibles. Con este fin, una vez planteado brevemente el problema en este apartado introductorio, en el segundo se incide en las dificultades específicas para definir, delimitar y cuantificar el sector. En el tercer apartado se clasifican y describen las principales bases de datos referidas directa o indirectamente al sector de la restauración en España y, en el cuarto, se analizan y comparan las bases de datos oficiales, ilustrando las principales diferencias entre ellas a través de la confrontación de datos referidos a demografía empresarial y a la dimensión económica del sector. El artículo finaliza con un apartado donde se recogen las principales conclusiones y se plantean algunas sugerencias para mejorar la información estadística y contribuir al análisis y mejor comprensión del sector. 


\section{Dificultades para definir y cuantificar el sector de la restauración en España}

Definir y delimitar el sector de la restauración no es sencillo. En este trabajo nos referiremos a la restauración en sentido amplio, definiéndolo como «el conjunto de establecimientos abiertos al público cuya actividad principal consiste en proveer servicios de comidas y/o bebidas», abarcando por tanto algo más que restaurantes, pero no la hostelería en su conjunto, que englobaría alojamiento más comida. No obstante, dependiendo del objeto de estudio y de la disponibilidad de estadísticas en cada momento, dado que la provisión de comidas y bebidas puede tener lugar en establecimientos cuya actividad principal no sea esta, como por ejemplo en los hoteles, podría ser oportuno ampliar el concepto. En estos casos, lo ideal sería capturar el valor correspondiente a la provisión de comidas y/o bebidas y desgajarlo del valor del resto de bienes y/o servicios provistos, algo que no siempre es posible. Por otra parte, también es preciso reconocer que ni aun en sentido más restringido hay total consenso en cuanto a la delimitación de establecimientos considerados propios de la restauración, por ejemplo, acerca de si las máquinas expendedoras de comidas y bebidas (vending) deberían o no ser objeto de inclusión en el sector.

Hablar de restauración conduce inevitablemente a pensar en la actividad conocida como comer fuera del hogar. Así como el hecho de comer fuera de casa constituye en gran medida un fenómeno cultural, la propia definición está, irremediablemente, impregnada de tintes culturales. A modo de ejemplo, las respuestas espontáneas obtenidas en la encuesta Enhali-2012, cuyo cuestionario deja en manos del entrevistado la definición de sus límites, traducen que, mientras para unos es comer en un restaurante, para otros lo es hacerlo en casa de amigos o incluso llevar la comida preparada en casa para comerla fuera (Díaz Méndez y García Espejo, 2013). De aquí podríamos deducir que, en España, el concepto comer fuera de casa está claramente marcado por el hecho físico de comer fuera, en un espacio ajeno a nuestra propia vivienda. Sin embargo, en el mundo anglosajón eating out se asimila más bien al hecho pragmático de comer - dentro o fuera del hogar - algo no preparado en casa, siendo por tanto más factible que el sector de la restauración resulte definido en este sentido con límites más amplios, incluyendo cualquier tipo de servicio que permita llevarse/encargar comida para comer en casa. Así, en este contexto, el mercado de food service podría incluir cualquier tipo de canal, desde los consumos realizados en el hogar de productos preparados y listos para comer adquiridos en restaurantes, pasando por pedidos fuera/envíos a domicilio, hasta las compras efectuadas en máquinas expendedoras o incluso en supermercados/ hipermercados o tiendas de alimentación tradicional. Esta es precisamente la delimitación aplicada por la consultora internacional NPD en su panel CREST (Consumer Reports on Eating).

En definitiva, la definición de restauración parece estar mediatizada por el contexto sociocultural en el que resulte planteada, así como por la interpretación que al concepto de comer fuera del hogar se dé en cada caso y viceversa. 
Así, si lo que se pretende es analizar el sector de la restauración, como es nuestro caso, el concepto comer fuera del hogar ya no incluiría, por ejemplo, comer en casa de unos amigos o de algún familiar, ni el hecho de llevarse la comida preparada en casa para comérsela en un parque o en el comedor de una empresa, asimilándose al concepto food service, con límites más o menos amplios, pero - en cualquier caso- en relación con comidas y/o bebidas preparadas fuera del hogar y listas para su consumo inmediato, mediando una transacción económica a cambio de tal servicio.

La restauración se ha convertido en un sector clave en la economía española, en parte debido a la importancia que el turismo ha ido adquiriendo en nuestra economía y, quizás en mayor medida, a los cambios socioeconómicos y los nuevos comportamientos sociales que vienen aconteciendo desde los años 60 , que han contribuido a que comer o cenar fuera del hogar sea una actividad generalizada entre los españoles desde hace algunas décadas. A pesar de ello, se detectan importantes lagunas y limitaciones en la información disponible para su cuantificación, análisis y comprensión. Más allá de las cuestiones ya señaladas respecto a los servicios en general, la propia naturaleza de los servicios de restauración complica aún más esta precariedad. Entre otros, cabe destacar los siguientes rasgos: se trata de un sector dinámico, con una oferta muy heterogénea de servicios; tiene un fuerte carácter estacional, aspecto que dificulta mantener plantillas fijas regulares; es heterogéneo en sus necesidades de empleo (con la confusión añadida entre asalariados fijos discontinuos, eventuales y no asalariados); está formado por numerosas empresas, generalmente de pequeño tamaño, en algunos casos con la posibilidad de llevar a cabo diferentes actividades en la misma unidad de explotación, del mismo modo que empresas con una actividad principal distinta (como es el caso de los hoteles y similares) prestan servicios asociados de restauración; y, por último, soporta un gran peso de la economía sumergida (Figuerola, 2013; Figuerola, 2015; García y Pin, 2010).

\section{La dispersión de estadísticas y las principales bases de datos disponibles}

A pesar de los avances comentados previamente, la información disponible sobre restauración en España adolece de importantes limitaciones. La heterogeneidad y dispersión del sector, así como la aparente ausencia de interés en elaborar un sistema de información estadística integrado, redundan en la inexistencia de una base de datos específica, de modo que los datos se encuentran dispersos. Entre las distintas fuentes disponibles para estudiar directa o indirectamente el sector de la restauración en España, cabe diferenciar, según su origen, entre las oficiales y las de carácter privado. Entre las primeras, destacan las que provienen del INE y las procedentes del Ministerio de Agricultura, Alimentación y Medio Ambiente (Magrama). Véase la tabla 1.

A grandes rasgos, se detectan tres grandes diferencias entre las fuentes de información oficiales. En primer lugar, cabe reseñar que las bases de datos del INE están planteadas y elaboradas, fundamental aunque no exclusivamente, 
Tabla 1. Bases de datos procedentes de fuentes oficiales, para estudiar directa o indirectamente el sector de la restauración en España.

\begin{tabular}{|c|c|c|}
\hline Fuente & Registros/operaciones estadísticas & Enfoque \\
\hline \multirow{13}{*}{$\begin{array}{l}\text { INE (Instituto Nacional } \\
\text { de Estadística) }\end{array}$} & Directorio Central de Empresas (Dirce) & Oferta \\
\hline & Demografía Armonizada de Empresas (DAE) & Oferta \\
\hline & $\begin{array}{l}\text { Encuesta Estructural de Empresas del Sector Servi- } \\
\text { cios (EEESS). Antigua Encuesta Anual de Servicios }\end{array}$ & Oferta \\
\hline & Indicadores de Actividad del Sector Servicios (IASS) & Oferta \\
\hline & Encuesta de Población Activa (EPA) & Oferta \\
\hline & Encuesta trimestral de coste laboral & Oferta \\
\hline & Encuesta anual de coste laboral & Oferta \\
\hline & Encuesta cuatrienal de estructura salarial & Oferta \\
\hline & $\begin{array}{l}\text { Índice de Precios de Consumo (IPC), subclases, e } \\
\text { Índice de Precios de Consumo Armonizado (IPCA), } \\
\text { subíndices }\end{array}$ & Oferta y demanda \\
\hline & Cuenta Satélite del Turismo (CST) & Oferta y demanda \\
\hline & $\begin{array}{l}\text { Contabilidad Nacional de España: Tablas de Origen } \\
\text { y Destino (TOD). }\end{array}$ & Oferta y demanda \\
\hline & $\begin{array}{l}\text { Contabilidad Nacional de España: Clasificación del } \\
\text { gasto en consumo final de los hogares por finalidad } \\
\text { (COICOP) }\end{array}$ & Demanda \\
\hline & Encuesta de Presupuestos Familiares (EPF) & Demanda \\
\hline \multirow{6}{*}{$\begin{array}{l}\text { Magrama (Ministerio de } \\
\text { Agricultura, Alimenta- } \\
\text { ción y Medio Ambiente) }\end{array}$} & Panel del Consumo Alimentario Fuera del Hogar: & Demanda \\
\hline & $\begin{array}{l}\text { Estudio consumo alimentario extradoméstico en } \\
\text { España: Hábitos del consumidor y los informes Ten- } \\
\text { dencias de consumo fuera del hogar }\end{array}$ & \\
\hline & Panel NPD-CREST: & Demanda \\
\hline & $\begin{array}{l}\text { Estudio sobre el mercado extradoméstico de } \\
\text { alimentación. Principales variables que caracterizan } \\
\text { la demanda extradoméstica de alimentación desde } \\
\text { el punto de vista del consumidor }\end{array}$ & \\
\hline & $\begin{array}{l}\text { Panel del Consumo Alimentario Fuera del Hogar/ } \\
\text { Panel de Establecimientos del Sector Horeca: }\end{array}$ & Oferta \\
\hline & $\begin{array}{l}\text { Estudio consumo alimentario extradoméstico en } \\
\text { España: Sector Hostelería/Restauración }\end{array}$ & \\
\hline
\end{tabular}

Fuente: elaboración propia.

desde la perspectiva de la oferta, mientras que las procedentes del Magrama siguen predominantemente un enfoque de demanda, ya que aportan sobremanera datos sobre consumo, gasto y canales de compra. En segundo lugar, a diferencia de la información facilitada por el Magrama, respecto a la información provista por el INE, no se ha encontrado ninguna fuente específicamente centrada en el sector de la restauración, si acaso, bases de datos referidas al sector servicios, incluidos los correspondientes a comidas y bebidas. Por último, la acotación del subsector objeto de estudio responde a criterios distintos. 
Tabla 2. Cambio de la CNAE 93 a la CNAE 2009: impacto en las divisiones (dos dígitos) y grupos (tres dígitos) relacionados con la hostelería y la restauración

\begin{tabular}{lc}
\hline CNAE 93 & CNAE 2009 Rev. 2 \\
\hline 55: Hostelería & 55: Servicios de alojamiento \\
551: Hoteles & 56: Servicios de comidas y bebidas \\
552: Camping y otros tipos de hospedaje & 561: Restaurantes y puestos de comidas \\
de corta duración & 562: Provisión de comidas preparadas para \\
553: Restaurantes & eventos y otros servicios de comidas \\
554: Establecimientos de bebidas & 563: Establecimientos de bebidas \\
555: Comedores colectivos y provisión & \\
de comidas preparadas &
\end{tabular}

Fuentes: INE (www.ine.es/dyngs/INEbase/es/operacion.htm?c=Estadistica_C\&cid=1254736177032\&menu =ultiDatos\&idp=1254735976614) y EUROSTAT (http://ec.europa.eu/eurostat/documents/3859598/5902521/ KS-RA-07-015-EN.PDF).

El criterio de clasificación predominante en el caso de la información facilitada por el INE gira en torno a la CNAE (Clasificación Nacional de Actividades Económicas), a partir de la nomenclatura estadística de actividades económicas de la Comunidad Europea (NACE), si bien la Encuesta de Presupuestos Familiares (EPF) sigue el sistema de clasificación de Bienes y Servicios COICOP (Classification of Individual Consumption According to Purpose); una adaptación de la clasificación usada por Eurostat para las encuestas de presupuestos. Por su parte, la mayor parte de la información facilitada por el Magrama acerca del sector se refiere al denominado canal Horeca (Hoteles/Restaurantes/Cafés) más, en ocasiones, algunos comedores colectivos/catering, y no se contempla en ningún caso el consumo de no residentes.

Es preciso resaltar que, en el marco de la CNAE, ha tenido lugar un cambio metodológico potencialmente relevante para el objeto de estudio que nos ocupa, con la entrada en vigor en 2008 de la nueva clasificación de actividades. Tal y como se puede ver en la tabla 2, el paso de CNAE 93 a CNAE 2009 implicó, a nivel de dos dígitos, desagregar la división 55 (hostelería) en las nuevas divisiones 55 (servicios de alojamiento) y 56 (servicios de comidas y bebidas). No obstante, en numerosas ocasiones el INE solo difunde información referida a hostelería (léase la suma de las divisiones 55 y 56 CNAE 2009), como es el caso de las cuentas anuales de la CN (Contabilidad Nacional), bases 2008 y 2010. Al igual que el grupo 551 (hoteles) de la CNAE 93 comprendía servicios asociados de restauración, la división 55 de la nueva clasificación incluye servicios de comidas y bebidas provistos en hoteles y alojamientos similares (Eurostat, 2007). Respecto a los canales de distribución contemplados para acotar los servicios de comidas y bebidas, si bien se resalta que «lo decisivo es que se ofrezcan comidas listas para su consumo inmediato, y no el tipo de instalación que presta el servicio», se excluye expresamente la venta al por menor a través de máquinas expendedoras (Eurostat, 2007), tal y como se 
venía haciendo en la CNAE 93. En resumen, además del subsector comercial (restauración tradicional y moderna, como los restaurantes de comida rápida), tienen cabida las actividades de restauración asociadas al transporte, así como a comedores colectivos (explotación de cantinas o cafeterías en oficinas, fábricas, escuelas u hospitales), es decir, lo que algunos autores denominan subsector institucional o mercado cautivo (véase, por ejemplo, Romero, 2008).

En cuanto a las bases de datos del Magrama, cabe destacar la información procedente del Panel de Consumo Alimentario Fuera del Hogar, donde resaltan dos ámbitos de estudio: el referido a hábitos del consumidor y tendencias de consumo fuera del hogar, y el dedicado al «sector Horeca». Los estudios del primer ámbito vienen realizándose desde 2007 a través de 14.000 encuestas al semestre en las que colaboran 28.000 personas anualmente, con lo que se convierten en una de las principales bases de datos disponibles en España para el estudio de la alimentación desde la perspectiva de la demanda. Desde esta óptica, también es de mucha utilidad el Estudio sobre el mercado extradoméstico de alimentación. Principales variables que caracterizan la demanda extradoméstica de alimentación desde el punto de vista del consumidor, realizado por la empresa pública Mercasa, por encargo del Ministerio, con apoyo en el panel NPD-CREST. Se viene llevando a cabo desde 2007, con apoyo en una muestra de 120.000 individuos al año y se refiere al consumo de alimentos fuera del hogar de la población residente en España.

En relación con el Panel de Consumo Alimentario Extradoméstico referido al sector Horeca, los estudios se iniciaron en 1987, con apoyo en las siguientes encuestas: Encuesta en Hostelería-Restauración y Encuesta en Restauración Social-Instituciones. Desde 2007, recogen trimestralmente información sobre las compras efectuadas por 1.500 establecimientos Horeca (incluyendo hoteles) y 300 establecimientos de restauración social (en sanidad, educación, residencias de ancianos...), en particular, referida a cantidades, precios unitarios, gasto y lugar donde se ha realizado la compra. Frente a la encuesta en hogares, el gasto aquí se refiere al efectuado por el establecimiento proveedor del servicio en la adquisición de una parte representativa de los consumos intermedios en su proceso de producción y no al gasto realizado por el consumidor final. Permite capturar información desde la perspectiva de la oferta, referida por ejemplo a fuentes de compra de los establecimientos de la hostelería comercial, diferenciando por tipos de establecimientos.

El Anuario de Estadistica del Magrama ofrece información similar, y recoge las cuotas de mercado según los canales de compra en hostelería-restauración en su conjunto, sin diferenciar por tipos de establecimientos. Estas compras que los establecimientos del sector Horeca efectúan a otros (sub)sectores vienen a representar los consumos intermedios recogidos en la CN. Si bien se relacionan con la dimensión del sector, no constituyen el indicador clave, asociado a producción/ventas o al valor añadido. Sí constituyen, en cambio, el eslabón imprescindible para relacionar ambos conceptos, en la medida en la que para estimar el valor añadido de un sector es preciso restar los consumos intermedios al valor de su producción. 
Por último, conviene hacer un breve apunte acerca de las fuentes de información de carácter privado. En este ámbito destacan los informes que sistemáticamente viene publicando la FEHR en los últimos años (titulados Los sectores de la hostelería, ya en su 20 a edición) y el Anuario Económico de España, elaborado por La Caixa hasta 2013, que presentaba la ventaja de ofrecer información a nivel municipal, destacando, con vistas a nuestro objeto de estudio, los indicadores referidos a establecimientos o locales y el índice de restauración y bares, ambos apoyados en el impuesto de actividades económicas. Igualmente, existen distintos informes elaborados por consultoras como AC Nielsen (a cargo del mencionado Estudio consumo alimentario extradoméstico en España: Sector Hostelería/Restauración, de Magrama); DBK; Euromonitor International o la española Alimarket.

\section{Una aproximación al sector de la restauración a través de la estadística oficial}

En este apartado se confrontan datos procedentes de distintas fuentes oficiales, referidos a la demografía empresarial (número de empresas y de locales) y a la dimensión económica del sector, aproximada a través del volumen de producción (perspectiva de la oferta) o del gasto (perspectiva de la demanda), aspectos que nos permitirán no solo analizar y comparar las bases de datos oficiales, sino también acercarnos empíricamente al sector.

\subsection{Demografia empresarial}

El INE facilita información sobre el número de empresas y/o establecimientos en cuatro bases de datos distintas: Directorio Central de Empresas (Dirce); Demografía Armonizada de Empresas (DAE); Encuesta Estructural de Empresas del Sector Servicios (EEESS; antigua Encuesta Anual de Servicios) y la Cuenta Satélite de Turismo (CST). Esta última solo facilita datos referidos a empresas hasta el año 2008 inclusive y, en todo caso, remite al Dirce como única fuente de información. El último año para el que la información está disponible en todas las demás fuentes es 2015. Los datos correspondientes se recogen en la tabla 3.

A grandes rasgos, se detectan diferencias significativas entre las cifras arrojadas por las diferentes bases de datos. Veamos algunas posibles explicaciones. La razón de la diferencia entre Dirce y DAE es, básicamente, de carácter metodológico: esta última, con apoyo en la metodología acordada en el marco de la UE (Unión Europea), realiza una explotación estadística armonizada de los directorios del Dirce, tomando como período de referencia el año natural, mientras que el Dirce elabora los mencionados directorios con apoyo en numerosas fuentes de información (tributarias, Seguridad Social, jurídicas, estadísticas y privadas, entre otras), adoptando como período de referencia el primer día de cada año para las empresas económicamente activas. 
En resumen, DAE ofrece la ventaja de estar armonizada con otras bases de datos europeas, lo que permite las comparaciones internacionales, pero aporta menos información y con menor grado de desagregación (no facilita información sobre locales, por ejemplo) y, además, se actualiza con más rezago (el último dato publicado corresponde a 2015, frente al último año disponible en el Dirce, 2017). A su vez, la muestra para ejecutar la EEESS procede del Dirce (en lo que a empresas se refiere), mientras que la información solicitada en la encuesta acerca del número de establecimientos se asocia a una fecha, en concreto, el 30 de septiembre (y no al período de referencia del año natural, adoptado en general en la encuesta).

Por otra parte, desde el año 2008, las tres fuentes mencionadas se apoyan en la CNAE 2009. En concreto, en cuanto a demografía empresarial, el Dirce y la antigua Encuesta Anual de Servicios, a nivel de tres dígitos, y la DAE, a nivel de dos. Desde 2015, la EEESS sustituye a la Encuesta Anual de Servicios y facilita información a nivel de cuatro dígitos. La nueva clasificación CNAE 2009 no evita que parte de la información en lo que a demografía empresarial se refiere, de nuevo, quede fuera del sector de la restauración: desde 2008, la división 56, Servicios de comidas y bebidas, deja fuera los vinculados a algunos servicios de alojamiento, por ejemplo, los restaurantes de los hoteles y similares, tal y como venía ocurriendo previamente (en la CNAE 93, a nivel de tres dígitos, bajo el rótulo Hoteles se mezclaba, en su caso, alojamiento y comidas, véase la tabla 2). En suma, las cifras recogidas en la tabla 3 no dejan de ser una estimación a la baja del número de empresas y establecimientos del sector de la restauración en España.

Por último, dada la relevancia del Dirce como marco de referencia para otras estadísticas del INE, se está realizando un esfuerzo adicional en términos de complementar el análisis estático con un análisis dinámico, a efectos de mejorar los procesos de actualización de datos. En este contexto resulta clave considerar el fenómeno de las «reactivaciones» de las unidades poblacionales; ceses temporales de la actividad de una empresa, debidos, por ejemplo, a su carácter estacional o a bajas temporales de empresarios individuales. Pues bien, el INE ha detectado que la mayoría de las empresas reactivadas responden al

Tabla 3. Número de empresas y locales correspondientes a servicios de comidas y bebidas (división 56, CNAE 2009) en distintas bases de datos del INE. Año 2015

\begin{tabular}{ll}
\hline Dirce (Directorio Central de Empresas) & $\begin{array}{l}252.354 \text { empresas* } \\
274.638 \text { locales }\end{array}$ \\
\hline DAE (Demografía Armonizada de Empresas) & 287.607 empresas \\
\hline EEESS (Encuesta Estructural de Empresas & $\begin{array}{l}247.036 \text { empresas } \\
\text { del Sector Servicios) }\end{array}$ \\
\hline CST (Cuenta Satélite de Turismo) & Datos disponibles solo hasta 2008 y remite al Dirce \\
\hline *253.344 a 1 de enero de 2017 & \\
Fuente: elaboración propia a partir de datos del INE.
\end{tabular}


Tabla 4. Dimensión económica del sector de la restauración en España. Año 2015, en millones de euros.

\begin{tabular}{|c|c|c|c|c|}
\hline Operaciones estadísticas & Fuente & Enfoque & Sistema de clasificación & Producción/gasto \\
\hline $\begin{array}{l}\text { EEESS (Encuesta Estructural de } \\
\text { Empresas del Sector Servicios, } \\
\text { antigua Encuesta Anual de } \\
\text { Servicios) }\end{array}$ & INE & $\begin{array}{l}\text { Oferta, cifras refe- } \\
\text { ridas a producción }\end{array}$ & $\begin{array}{l}\text { CNAE (Clasificación Nacional de } \\
\text { Actividades Económicas) } 2009\end{array}$ & 42.113 \\
\hline $\begin{array}{l}\text { Contabilidad Nacional de Espa- } \\
\text { ña: TOD (Tablas de Origen y } \\
\text { Destino). Base } 2008\end{array}$ & INE & $\begin{array}{l}\text { Oferta, cifras refe- } \\
\text { ridas a producción }\end{array}$ & $\begin{array}{l}\text { CNAE } 2009 \text { y CPA (Clasificación } \\
\text { de Productos por Actividad) } \\
2008\end{array}$ & $92.559^{\star}$ \\
\hline $\begin{array}{l}\text { Contabilidad Nacional de } \\
\text { España: Gasto en consumo } \\
\text { final de los hogares. Base } 2010\end{array}$ & INE & $\begin{array}{l}\text { Demanda, cifras } \\
\text { referidas a gasto }\end{array}$ & $\begin{array}{l}\text { COICOP (Classification of Indivi- } \\
\text { dual Consumption According to } \\
\text { Purpose, clasificación del gasto } \\
\text { en consumo final de los hogares } \\
\text { por finalidad, de las Naciones } \\
\text { Unidas) }\end{array}$ & $92.008^{\star \star \star}$ \\
\hline $\begin{array}{l}\text { Encuesta de Presupuestos } \\
\text { Familiares (EPF). Base } 2006\end{array}$ & INE & $\begin{array}{l}\text { Demanda, cifras } \\
\text { referidas a gasto }\end{array}$ & COICOP & 43.546 \\
\hline $\begin{array}{l}\text { Panel NPD-CREST (Consumer } \\
\text { Reports on Eating) }\end{array}$ & Magrama & $\begin{array}{l}\text { Demanda, cifras } \\
\text { referidas a gasto }\end{array}$ & $\begin{array}{l}\text { No se aplica más criterio que } \\
\text { la exclusión de: no residentes, } \\
\text { instituciones y consumos en los } \\
\text { que no medie una transacción } \\
\text { económica directa }\end{array}$ & 31.994 \\
\hline
\end{tabular}

* Correspondiente a 2009, debido a que, desde 2010, la información difundida por el INE a partir de la Contabilidad Nacional Base 2010 presenta conjuntamente servicios de comidas y bebidas y de alojamiento. En cambio, la cifra aquí presentada incluye toda la producción correspondiente al concepto de comidas y bebidas con independencia de la rama de actividad donde fue generada (incluye, por ejemplo, las comidas servidas en los hoteles, sin mezclar con el valor de los servicios de alojamiento).

** La clase 11.1 (restaurantes, bares y cafeterías) recoge toda la actividad correspondiente a la hostelería, con independencia de los canales, exceptuando servicios de restauración en hoteles y otros alojamientos, si van incluidos en el precio del alojamiento (por ejemplo, bed and breakfast) y los alimentos y bebidas provistos a enfermos hospitalizados.

*** Estimación provisional.

Fuente: elaboración propia, con apoyo en las fuentes citadas.

perfil legal de una persona física y, tras esta forma jurídica, no ha de extrañar que sean la hostelería y la restauración las actividades con mayor presencia del fenómeno (véase INE, 2015). Lo indicado previamente pone de manifiesto que las características de las empresas del sector de la restauración conllevan mayor dificultad añadida a la hora de estudiar su evolución en el tiempo.

\subsection{Dimensión económica (producción y gasto)}

La dimensión económica de un sector puede valorarse desde la perspectiva de la oferta (por ejemplo, a través de cifras de la producción) o de la demanda (gasto o consumo). En esta sección realizaremos una primera aproximación a la dimensión económica de la restauración en España a través de las cifras obtenidas de cinco operaciones estadísticas distintas, dos referidas a la pro- 
ducción y tres al gasto. En la tabla 4 se recogen las cifras correspondientes al año 2015.

Antes de analizar las notables diferencias entre las cifras recogidas en la tabla 4 , es preciso resaltar que la obtenida de las TOD (Tablas de Origen y Destino) corresponde al año 2009, porque desde 2010 las TOD recogen conjuntamente los servicios de comidas y bebidas y los servicios de alojamiento, es decir, el INE deja de difundir esta información de forma desagregada. En la misma línea, se ha optado por prescindir de las CST debido a que desde 2008 (base 2008) el INE deja de difundir información al nivel de desagregación que aquí se precisa (por ejemplo, los valores de la producción de la rama Restaurantes y similares y otros aspectos tan relevantes como el peso que la parte turística tiene en la producción de servicios de comidas y bebidas). La otra cifra asociada al enfoque de la oferta es la referida a la producción obtenida a partir de la EEESS, siendo 2015 el último año disponible; de ahí, el año de referencia adoptado para la información recogida en la tabla 4 . De hecho, la cifra aportada en la introducción de este trabajo en relación con la producción del sector en 2016 (93.000 millones de euros) es en realidad una proyección llevada a cabo en Figuerola, Sánchez y Herranz (2017).

La discordancia entre las cifras recogidas en la tabla 4 se explica, en cierta medida, por cuestiones metodológicas. Por una parte, el hecho de seguir la perspectiva de la oferta o de la demanda implica el manejo de conceptos distintos (producción o gasto), de modo que, por definición, las cifras no deberían coincidir. Así, la producción se valora siguiendo la perspectiva del productor, por los costes totales de producción, incluyendo los consumos intermedios (no es solo valor añadido, a diferencia del gasto en consumo final) y a precios básicos, frente a la presentación de los datos a precios de venta al público (caso de la demanda, incluyendo por ejemplo el IVA). Véase Parlamento Europeo y Consejo, 2013. Por otra parte, más allá de las diferencias derivadas de la perspectiva seguida, conviene explicar las discordancias existentes entre los valores obtenidos desde una misma perspectiva.

Desde la perspectiva de la demanda, tanto la EPF (Encuesta de Presupuestos Familiares, INE) como el Panel NPD-CREST, del que parten muchos de los análisis del gasto alimentario fuera del hogar del Magrama, solo se refieren al gasto efectuado por ciudadanos residentes en España, de forma que queda por tanto excluido el gasto correspondiente a consumo de viajeros extranjeros en territorio español (turismo receptor) y de inmigrantes irregulares. A partir de ahí, es preciso explicar por qué la cifra extraída de la EPF, referida al subgrupo 111 Comidas y bebidas fuera del hogar, es superior a la derivada del Panel NPD-CREST (43.546 millones de euros frente a 31.994 millones de euros). Posiblemente, la explicación resida en que este último obvia los consumos en los que no media una transacción económica directa, por ejemplo, comedores escolares o celebraciones, que sí incluye la EPF, así como los consumos realizados en instituciones y que tampoco son contemplados en la EPF si se trata del consumo de residentes en hogares colectivos o instituciones, como cárceles o residencias de ancianos. Sí se consideran, en cambio, los servicios de comedor 
provistos por empresas y oficinas públicas a sus empleados a un precio inferior al de mercado.

Asimismo, llama la atención la gran distancia que separa las cifras de la EPF y del Panel NPD-CREST de las correspondientes al gasto en consumo final correspondiente de la CN de España, cuya rúbrica, con 92.008 millones de euros, más que duplica las mencionadas previamente. Parte de esta diferencia responde a que el gasto en consumo final de los hogares de la CN que el INE presenta por divisiones incluye el efectuado por ciudadanos no residentes en España (lo descuenta después, pero sin especificar de qué divisiones procede, así que no es posible estimar por esta vía el peso que el turismo receptor representa para el sector de la restauración). Por otra parte, dado que las estimaciones de la EPF son usadas para calcular el consumo privado en la CN, el propio INE recoge algunas posibles explicaciones de las brechas que separan las cifras de la CN y la EPF: como los criterios de valoración, los momentos del registro o el hecho de que en la primera se incluyan los establecimientos colectivos, mientras que la EPF tiene en cuenta solo los consumos de residentes en hogares privados (véase INE, s. f.). Otra posible razón de la diferencia que separa las cifras arrojadas por la EPF y la CN podría relacionarse con la solicitud de la declaración de ingresos que acompaña a la EPF. Así, en la medida en la que «para el encuestado sus respuestas quedan grabadas, para un ente estatal, simbólicamente vinculado con Hacienda y la política impositiva», es muy probable que las cifras declaradas de ingresos y gastos resulten sesgadas a la baja (Díaz Méndez et al. 2005).

De lo indicado previamente se desprende que, con las cifras del Magrama en la mano, el peso del gasto alimentario extradoméstico frente al gasto alimentario en el hogar (alrededor de un tercio frente a dos tercios del gasto total) resulte inferior a si se toman los datos del consumo final de los hogares de la $\mathrm{CN}$.

Desde la óptica de la oferta, las cifras referidas a la producción del sector recaban información sobre la provisión de servicios de restauración desde la perspectiva empresarial, con independencia de quién sea el sujeto consumidor de los mismos, es decir, a diferencia de la mayoría de las encuestas dirigidas a la demanda, incluyen por ejemplo el valor de los servicios prestados al turismo receptor. Además, en el caso español, las cifras asociadas al enfoque de la oferta incluyen los establecimientos colectivos. De ahí que sea sorprendentemente baja la cifra que arroja la EEESS. Es cierto que, siguiendo la clasificación CNAE 2009, por la que se rige la EEESS, la división 56, tal y como se expuso previamente, deja fuera los servicios de comidas y bebidas provistos en hoteles y similares, lo que no ocurre con las TOD, que permiten recabar el valor de la actividad correspondiente, con independencia de la rama de actividad donde fue generada. No obstante, la enorme brecha que separa las cifras correspondientes parece responder más bien a ocultación de ventas en la EEESS y, en definitiva, al peso de la economía sumergida en el sector. De hecho, la EEESS es una base de datos muy criticada en este sentido de forma recurrente en los informes anuales de la FEHR (véase, por ejemplo, Figuerola, 2015). 
En resumen, entre las posibles razones de la discordancia de las cifras recogidas en la tabla 4, destacan cuestiones metodológicas, relacionadas tanto con la perspectiva adoptada como con los canales de distribución contemplados en cada caso; posibles sesgos a la baja («cifras contaminadas» por su asociación por los entrevistados con las autoridades fiscales), y ocultación de ventas, reflejo del peso de la economía sumergida en el sector.

Por último, cabe referirse a los informes estadísticos que viene realizando Eurostat, con análisis de demografía empresarial, estructurales o por regiones y países. Lamentablemente, en los análisis por países la hostelería se trata como un único sector. El último informe disponible (Eurostat, 2017) señala que para el conjunto de la UE-28, en 2014, el sector de la provisión de comidas y bebidas (el 56) contabiliza el $84,2 \%$ de las empresas de hostelería, el 77,2\% del personal empleado y el 66,1\% del valor añadido. Eso sí, con un nivel de productividad por trabajador que resulta ser el menor de todos los subsectores no financieros de la clasificación CNAE.

\section{Conclusiones}

La restauración es uno de los sectores clave tanto del turismo como de la economía española. Sin embargo, su análisis se enfrenta a importantes limitaciones y lagunas estadísticas. Entre estas, cabe resaltar la escasez de información cuantitativa referida específicamente al sector y la existencia de incoherencias incluso entre datos oficiales procedentes de la misma fuente. Parte de estos problemas guarda relación con una tradición de apoyo oficial a las estadísticas hoteleras, de donde resulta que algunas estadísticas disponibles, en el mejor de los casos, acaben englobando la restauración y las actividades de alojamiento bajo el mismo paraguas.

El principal problema para estudiar el sector de la restauración no reside tanto en la falta de bases de datos como en la dispersión y heterogeneidad de los existentes y, sobre todo, en las incoherencias que no quedan plenamente explicadas por cuestiones metodológicas. En este sentido, es llamativo que existan discrepancias notorias entre cifras procedentes de la misma fuente, obtenidas desde la misma perspectiva y siguiendo el mismo sistema de clasificación: como el caso descrito para el enfoque de la oferta de las cifras obtenidas de la EEESS y de las tablas TOD, ambas procedentes del INE y apoyadas en el sistema de clasificación de la CNAE 2009. Respecto al enfoque de la demanda, las cifras del INE, referidas al gasto en consumo final de los hogares estimado en la CN y al obtenido en la EPF, resultan dispares, a pesar de compartir el sistema COICOP de clasificación del gasto. Más allá de cuestiones metodológicas (por ejemplo, diferencias conceptuales implícitas en el enfoque seguido o que, tal y como está planteado, el gasto de la $\mathrm{CN}$ es un concepto más amplio que el correspondiente a la EPF), otras razones parecen explicar las discrepancias: ocultación de ventas en el caso de la EEESS y sesgo a la baja en las cifras declaradas por los encuestados en la EPF. 
En consecuencia, si se pretende analizar el sector desde la óptica de la oferta, cabría dar prioridad a la información procedente de la CN-TOD, por ser cifras mucho más fiables que las derivadas de la EEESS, del mismo modo que, si la perspectiva a seguir es la demanda, parecen cifras más comprehensivas las que ofrece la $\mathrm{CN}$-gasto en consumo final de los hogares. Sin embargo, desde 2010, las TOD del INE dejan de presentar en rúbricas separadas los servicios de comidas y bebidas y los servicios de alojamiento.

La pertinencia de utilizar unas u otras fuentes dependerá de lo que pretendamos analizar en cada momento y, en consecuencia, del enfoque elegido. Si lo que se pretende es analizar la dimensión económica del sector, con independencia de quién gasta (en el sentido de si se trata o no de un residente en España), es preferible seguir el enfoque de la oferta. Nótese que el enfoque de la demanda prescinde de una parte importante de la actividad del sector en el ámbito turístico (en concreto, el turismo receptor, asociado a ciudadanos no residentes en España), así como de otros consumos que a pesar de haber sido realizados por residentes en establecimientos de restauración no fueron objeto de cómputo (fundamentalmente por no haber mediado una transacción económica directa). En consecuencia, si el foco de atención del estudio se dirige al sector de la restauración como sector económico, convendría analizarlo como mínimo- desde la perspectiva de la oferta, a efectos de no infravalorar su verdadera aportación a la economía.

Si bien las cifras manejadas y ofrecidas por el Magrama (correspondientes al gasto/compras efectuadas por los consumidores residentes en España en el sector de la restauración) tienden a infravalorar la verdadera dimensión económica del sector, la virtualidad del enfoque seguido en este tipo de encuestas reside en que permite analizar aspectos inabordables a partir de estadísticas obtenidas en el flanco de la oferta, como podrían ser los hábitos de los consumidores o sus motivaciones. De este modo, en el análisis del sector, en la medida de lo posible y con las debidas cautelas, conviene no perder de vista la posibilidad de combinar la información procedente del flanco de la oferta con la procedente del flanco de la demanda: tanto la EPF (INE) como el Panel de Consumo Alimentario (Magrama) aportan información sobre consumo de alimentos en cantidades físicas. En particular, el marco Panel del Consumo Alimentario Fuera del Hogar aporta, además, información acerca de las visitas, el perfil del consumidor, dónde, cuándo, con quién o por qué come fuera.

A modo de conclusión, el análisis del sector de la restauración en España a partir de las principales fuentes estadísticas oficiales disponibles se enfrenta a dos cuestiones particularmente preocupantes: las incoherencias entre bases de datos de un mismo organismo y el menor grado de desagregación que en los últimos años ofrecen las cuentas económicas de la CN de España del INE, en particular las TOD. Por lo tanto, frente a esta situación, cabe apuntar la necesidad de filtrar y depurar información contradictoria (algunos datos de la EEESS no parecen realistas) y de no perder grados de desagregación de la información difundida (como es el caso de la $\mathrm{CN}$ y de las CST). Asimismo, dada la relevancia del sector, sería deseable un esfuerzo de unificación y armo- 
nización de las bases de datos oficiales existentes, a efectos de facilitar información tan detallada como la disponible por ejemplo para los alojamientos.

\section{Referencias bibliográficas}

CASTRO, M. A. DE (2000). «Las estadísticas de turismo del INE en la actualidad (19942000)». Estudios Turísticos (144-145), 75-102. <http://estadisticas.tourspain.es/ img-iet/Revistas/RET-144-145-2000-pag75-102-84444.pdf>.

CUADRADO ROURA, J. R. (2003). «Expansión y dinamismo del sector servicios». Información Comercial Española (ICE): Revista de Economía (811), diciembre, 273-296. <http://www.revistasice.com/CachePDF/ICE_811_273-296_4CF526E28B068 6B1EA412340AF9E2C2A.pdf>.

Díaz Mendez, C.; Herrera, P., Callejo, J. y Alonso, L. E. (2005). «Análisis crítico de las fuentes estadísticas de consumo alimentario en España. Una perspectiva sociológica». REIS (Revista Española de Investigaciones Sociológicas) (110), 117-136. <https://doi.org/10.2307/40184685>.

Díaz MÉndeZ, C. y GARCía EsPejO, I. (2013). «La alimentación fuera del hogar». En: Díaz Méndez, C. (coord.). Hábitos alimentarios de los españoles, 47-61. Madrid: Ministerio de Agricultura, Alimentación y Medio Ambiente.

EUROSTAT (2007). «Estructura y notas explicativas». NACE Rev. 2. Eurostat - Unidad 02. Gobernanza estadística, calidad y evaluación. <http://www.ine.es/daco/daco42/ clasificaciones/cnae09/notas.pdf> (consultado el 14 de marzo de 2018).

- (2017). «Accomodation and food service statistics». NACE Rev. 2. <http:// ec.europa.eu/eurostat/statistics-explained/index.php/Accommodation_and_food_ service_statistics_-_NACE_Rev._2\#Structural_profile> (consultado el 14 de marzo de 2018).

Figuerola, M. (dir.) (2013). Los sectores de la hostelería en 2012. Madrid: FEHR (Federación Española de Hostelería). <http://www.fehr.es/documentos/publicaciones/descargas/des-79.pdfs.

- (2015). Los sectores de la hostelería en 2014. Madrid: FEHR (Federación Española de Hostelería). <http://www.fehr.es/documentos/publicaciones/descargas/des-92. pdf>.

Figuerola, M.; SÁnchez, M. y HerRanZ, J. L. (2017). Los sectores de la hostelería. 2016-2017. Madrid: FEHR (Federación Española de Hostelería). <http://www. fehr.es/documentos/publicaciones/descargas/des-102.pdf>.

GARCíA, P. y PIN, J. R. (2010). La rotación en el sector de la restauración en España. Causas, consecuencias y lineas de actuación. IRCO (International Research Center on Organizations) e InsedNetwork (International Network for Service Enterprise and Education Development). <http://www.iese.edu/Aplicaciones/upload/LBinsednetwork9_1.pdf>.

Guerra, J. L. (2009). «El sector de la restauración en España». Distribución y Consumo (103), enero-febrero, 32-39. <http://www.mercasa.es/files/multimedios/ pag_032-039_sector-guerra.pdf>.

INE (2015). Movimientos en el Dirce a 1 de enero de 2015. <http://www.ine.es/daco/ daco42/dirce/dirce15.pdf> (consultado el 15 de abril de 2016).

INE (s. f.). Encuesta de Presupuestos Familiares. Informes metodológicos estandarizados. $<$ http://www.ine.es/dynt3/metadatos/es/RespuestaDatos.html?oe=30458> (consultado el 21 de abril de 2016). 
Parlamento Europeo y Consejo (2013). «Reglamento (UE) no 549/2013 del Parlamento Europeo y del Consejo de 21 de mayo de 2013, relativo al Sistema Europeo de Cuentas Nacionales y Regionales de la Unión Europea». Diario Oficial de la Unión Europea L 174, de 26 de junio de 2013. <http://www.boe.es/ doue/2013/174/L00001-00727.pdf> (consultado el 21 de abril de 2016).

Romero, D. (2008). «Análisis del sector de la restauración en España: oferta y peso económico actual». Innovación y experiencias educativas, no 13 . <http://www.csicsif.es/andalucia/modules/mod_ense/revista/pdf/Numero_13/DAVID_ROMERO_1.pdf>. 
